\title{
iPhone/iPod Touch as Input Devices for Navigation in Immersive Virtual Environments
}

\author{
Ji-Sun Kim* \\ Virginia Tech, USA
}

\author{
Denis Gračanin ${ }^{\dagger}$ \\ Virginia Tech, USA
}

\author{
Krešimir Matković \\ VRVis, Austria
}

\author{
Francis Quek ${ }^{\S}$ \\ Virginia Tech, USA
}

\begin{abstract}
iPhone and iPod Touch are multi-touch handheld devices that provide new possibilities for interaction techniques. We describe iPhone/iPod Touch implementation of a navigation interaction technique originally developed for a larger multi-touch device (i.e. Lemur). The interaction technique implemented on an iPhone/iPod Touch was used for navigation tasks in a CAVE virtual environment. We performed a pilot study to measure the control accuracy and to observe how human subjects respond to the interaction technique on the iPhone and iPod Touch devices. We used the preliminary results to improve the design of the interaction technique.
\end{abstract}

Index Terms: I.3.6 [Methodology and Techniques]: Interaction techniques-; H.5.2 [User Interfaces]: Input devices and strategies-

\section{INTRODUCTION}

The acceptance and success of immersive virtual environments (IVEs) for navigation tasks strongly depends on a successful interaction technique. There are many approaches to navigation tasks in IVEs. Some approaches use physical locomotion of the user, such as treadmill walking, and some use a device, such as joystick. General acceptance of navigation interaction techniques that use some kinds of physical locomotion is assumed to be higher [2].

Recent introduction of iPhone [3] and iPod Touch opens new possibilities for interaction techniques on devices that have a multitouch screen. Multi-touch devices can sense multiple touches simultaneously [4]. Although the technique was available at least since mid 80's [8], it has not been used extensively until recently due to limited availability of devices that support it. Single-touch screens found their application at publicly accessible installations (such as museums, ATMs, tourist information kiosks, etc.) but did not find their way to the everyday use. Problems with precision, arm fatigue and response time are the largest hurdles. Introduction of iPhone or special devices such as ThinSight [5] made a large community aware of multitouch devices and their potential. These multitouch devices are used more and more in interaction design $[1,9]$. However, the task of designing a multitouch interaction is far from trivial. Effective interaction techniques are crucial for the success of new interaction paradigm.

\section{Proposed Interaction Technique}

In our previous work [7], we described a navigation interaction technique, named Finger Walking in Place (FWIP). Our goal was to take advantages of our inherent walking knowledge and use it for steering control with fingers. FWIP is divided into two parts, one for the translation of a viewpoint and one for the rotation of

\footnotetext{
*E-mail: hideaway@vt.edu

†E-mail: gracanin@vt.edu

‡E-mail: matkovic@ vrvis.at

${ }^{\S}$ E-mail: quek@vt.edu
}

a viewpoint, named "walking-in-place" and "rotation-in-place", respectively. For "walking-in-place", we use finger(s) that move as if human legs move on a tread mill. For "rotation-in-place", one finger is touching one spot without moving and another finger is dragging to the left or the right. A user can move in any direction, and change orientation in a virtual world. Virtual locomotion speed is controlled using the size and the frequency of finger movements. In order to evaluate FWIP for navigation tasks in IVEs, we used a Lemur device $[6]^{1}$ with the multitouch surface/screen size of 12 inch diagonally and resolution $800 \times 600$.

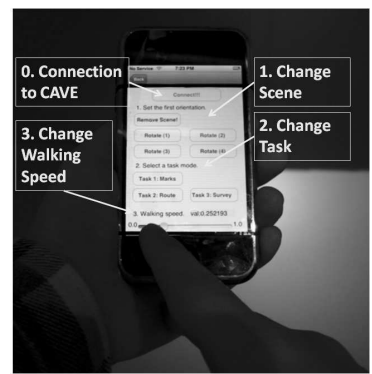

(a) Control mode

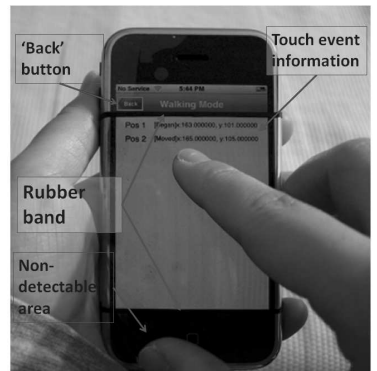

(b) Walking mode
Figure 1: User interfaces for iPhone/iPod Touch

The original one-handed FWIP developed for the Lemur device is modified because the touch information detected by iPhone/iPod Touch device is different from the one detected by the Lemur device (stationary touch event).

The modification is made only for the rotation technique because the rotation technique requires multiple touches. When using the Lemur device, a user simply keeps touching with the thumb while the index finger (or another finger the user prefers) is used for dragging. When using iPhone/iPod Touch, the user touches the screen with two fingers at the same time, drags both, and takes both of the screen at the same time.

For the experiment, we designed the user interfaces with two modes, the control mode for evaluators and the walking mode for human subjects. As shown in Figure 1(a), evaluators can control the system setup specific to the experiment. A human subject can control a viewpoint using the multitouch screen, as shown in Figure 1(b). In the pilot study, we observed that most of the subjects accidently touched the 'back' button or touch the non-detectable area while they are moving their fingers without looking at the screen. We attached the rubber bands to limit the walking areas on the iPhone screen (Figure 1(b)). Two components of FWIP for iPhone/iPod Touch are illustrated in Figure 2(a) "walking-inplace", and Figure 2(b) for "rotation-in-place".

\section{Pilot Study}

In order to evaluate FWIP on iPhone/iPod Touch, we conducted a pilot study using a CAVE installation. In this study, iPhone/iPod

${ }^{1}$ In July 2007, the company has adopted the name of Stantum (http://www.stantum.com), while JazzMutant remains the brand name for Lemur and other products under JazzMutant. 


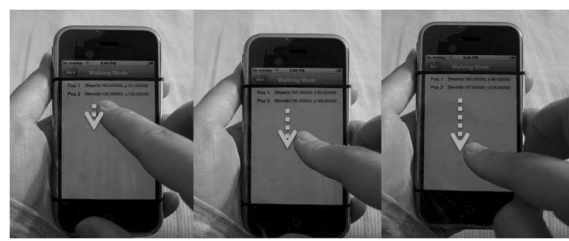

(a) Forward "walking-in-place"

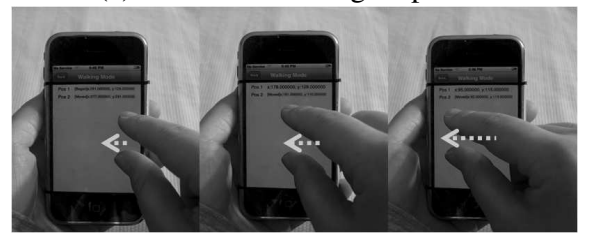

(b) Clockwise "rotation-in-place"

Figure 2: FWIP using iPhone/iPod Touch

Touch is only used as a surface for finger-walking. Navigation direction is only changed by finger movement. In order to conduct the experiment with the constraint that users would not physically move in the CAVE immersive space, we placed an iPhone/iPod Touch on the table to provide a persistent spatial reference (Figure 3).

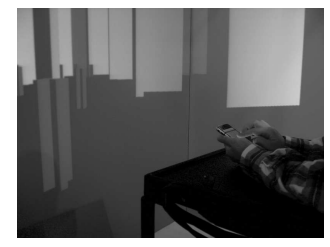

Figure 3: FWIP with iPhone/iPod Touch

This pilot study was conducted with the objective to determine how FWIP can be applied for a multitouch handheld device, such as iPhone, for navigation tasks in IVEs. We asked subjects to hold an iPhone/iPod Touch with the non-dominant hand, align it with the vertical line of the front wall in the CAVE space, and move their fingers with the dominant-hand on the screen surface. Our observation focuses on how subjects responded to FWIP using an iPhone/iPod Touch for navigation task in IVEs.

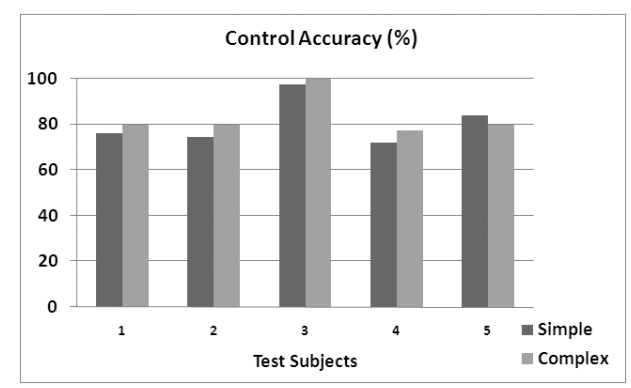

Figure 4: Control Accuracy

We used two virtual mazes. The simple and complex mazes sizes are approximately 245 by 195 units and 230 by 230 units, respectively. The cumulative angle to turn a viewpoint from one marker to another is larger in the complex maze (699 vs. 479 degrees). The route through the maze is presented with markers (i.e. cross-rectangular objects, Figure 3). The route length presented with marker objects is a little longer in the complex maze (377 vs. 310 units from the first to the last marker).
We did not provide any visual feedback except for the shadows of virtual objects. We measured how many marker objects subjects directly hit. This dependent variable was measured to investigate how accurately they can control the interface for the navigation task (Figure 4). The average hit rates in the simple and the complex mazes are $80.8 \%$ and $83.5 \%$, respectively. The results suggest that users may navigate with sufficient precision. Even though FWIP is already evaluated with a notebook size multitouch device (i.e. Lemur), we observed that some differences between Lemur and iPhone/iPod Touch may influence the task performance; (1) the walking surface area of iPhone/iPod Touch is smaller than Lemur; (2) the rotation technique is different from Lemur. Interestingly, two subjects tried to keep touching with thumb and only drag index finger on the iPhone surface (i.e. "rotation-in-place" for Lemur), even though they never used FWIP before; and (3) even though this is a one-handed technique, a subject should use the non-dominant hand to hold the iPhone, while the dominant hand is used for FWIP. We asked subjects to align the device with the front vertical line, but it is not strictly aligned, compared to the Lemur device that was fixed on the table.

In addition, two subjects commented that the touch sensitivity of iPhone/iPod Touch is too high, and they sometimes felt jumpy during navigation. We found it happened when they frequently made very short finger movement. FWIP implementation should be adjusted to finger movements on iPhone/iPod Touch.

\section{Conclusion}

In this paper, we described the implementation and presented some preliminary results of FWIP applied for iPhone/iPod Touch devices. FWIP was previously implemented for Lemur device but due to different sensing techniques and smaller touch surface we had to slightly modify the interaction technique.

Future work will proceed in two directions. First, we will use the results of our ongoing formative study to fine tune the proposed interaction technique. Second, we will perform a comparative study of several navigation interaction techniques with different input devices, including joystick, Lemur, and iPhone/iPod Touch. The results of the study should provide some guidelines about the use of multitouch devices for navigation and related tasks.

\section{REFERENCES}

[1] H. Benko, A. D. Wilson, and P. Baudisch. Precise selection techniques for multi-touch screens. In Proceedings of the SIGCHI conference on Human Factors in computing systems (CHI '06), pages 1263-1272, New York, 2006. ACM.

[2] D.A. Bowman, E. Kruijff, J.J. LaViola, Jr. , and I. Poupyrev. 3D User Interfaces: Theory and Practice. Addison-Wesley, Boston, 2004.

[3] S. Grissom. iPhone application development across the curriculum Journal of Computing Sciences in Colleges, 24(1):40-46, Oct. 2008.

[4] J. Han. The multi touch screen. http://multi-touchscreen.com/. Last accessed 27 Oct. 2008

[5] S. Hodges, S. Izadi, A. Butler, A. Rrustemi, and B. Buxton. ThinSight: Versatile multi-touch sensing for thin form-factor displays. In Proceedings of the 20th annual ACM symposium on User interface software and technology (UIST '07), pages 259-268, New York, 2007. ACM.

[6] JazzMutant. Lemur. http://www.jazzmutant.com/lemur_overview.php. Last accessed 30 Dec. 2008.

[7] J. Kim, D. Gračanin, K. Matković, and F. Quek: Finger Walking in Place (FWIP): A Traveling Technique in Virtual Environments. In Proceedings of 9th International Symposium, Smart Graphics 2008, pages $58-69,2008$

[8] S. Lee, W. Buxton, and K. C. Smith. A multi-touch three dimensional touch-sensitive tablet. ACM SIGCHI Bulletin, 16(4):21-25, Apr. 1985.

[9] T. Moscovich and J. F. Hughes. Multi-finger cursor techniques. In Proceedings of Graphics Interface 2006 (GI '06), pages 1-7, Toronto, Ont., Canada, 2006. Canadian Information Processing Society. 